\title{
Emergency alleviation of thermal overloads using model predictive control
}

\author{
B. Otomega Student, IEEE \\ A. Marinakis \\ M. Glavic Senior Member, IEEE \\ T. Van Cutsem, Fellow, IEEE
}

\begin{abstract}
An approach inspired of Model Predictive Control is proposed to determine a sequence of control actions aimed at alleviating thermal overloads in emergency conditions. The algorithm brings the line currents below their limits in the time interval left by protections, while accounting for constraints on control changes at each step. Its closed-loop nature allows to compensate for measurement noise and model uncertainties.
\end{abstract}

Index Terms-Thermal overload, emergency control, model predictive control, constrained optimization

\section{INTRODUCTION}

Some of the recent blackouts involved cascade line trippings due to thermal overloads. In some cases, emergency measures were not taken to quickly prevent some of the overloaded lines from being tripped, thereby leading to cascading effects on the remaining lines.

In such emergency conditions, it is essential to quickly mitigate the consequences of the initial disturbances before protection systems take actions that make the problem more severe [1]. As is well-known, protections may take the overloaded line out of service after some temporization or the line may sag and eventually touch objects, causing a short-circuit, quickly eliminated by distance protections.

Depending on the system, emergency control actions may involve changing the angle of phase shifting transformers, rescheduling generation and, in the last resort, shedding load. Topology changes and FACTS devices may also prove very efficient but are not considered here.

The controller proposed in this paper could be the heart of a system protection scheme [2]. To the authors' knowledge, there are very few system protection schemes in operation dealing with thermal overloads and cascade line trippings. One of the reasons is the need to rely on a system-wide model since line overloads can be relieved by acting on remote components, as opposed to underfrequency and undervoltage load shedding, for instance, which can rely on local signals and act locally.

An Optimal Power Flow (OPF) algorithm with proper objective and constraints can be used to determine the best actions. Many publications have been devoted to improving OPF algorithms, and OPF is available in Energy Management Systems [3], [4]. In many control centers, however, the OPF output is only proposed to the operator, who is responsible

\footnotetext{
The authors are with the Dept. of Electrical Engineering and Computer Science (Montefiore Institute) of the University of Liège, Sart Tilman B37, B-4000 Liège, Belgium. M. Glavic is invited professor and T. Van Cutsem (t.vancutsem@ulg.ac.be) is with the Belgian National Fund for Scientific Research (FNRS) at the same department.
}

for implementing the corresponding changes [5]. More importantly, this approach is static and open-loop by nature, which is not appropriate for the emergency problem under concern, as discussed hereafter.

Time is important in two, somewhat contradictory respects. On one hand, there is some time left to alleviate overloads, thanks to thermal inertia of equipments. Progress has been made in the real-time estimation of the time left before the conductor material is damaged or the line sag leaves insufficient insulation distance [6]. On the other hand, there are limits on the rate of change of some of the above mentioned controls: for instance, it takes time to change the tap position of a phase shifting transformer, the rate of change of power plant production is limited, etc.

To account for this, instead of a simultaneous change in all controls, a time sequence of control actions can be determined by solving a multi-step optimization problem.

However, this multi-step optimization would provide a single "optimal" control sequence for the available system model and the given initial condition. The open-loop nature of this optimization would not allow to compensate for inaccuracies originating from modelling uncertainties, measurement noises and unexpected reactions of some components. Instead, it is desirable to resort to closed-loop control, relying on system response in the course of applying corrective actions.

To this purpose, this paper proposes an optimization procedure that bears the spirit of Model Predictive Control (MPC). MPC is a class of algorithms to control the future behavior of a system through the use of an explicit model of the latter [7]. At each control step the algorithm computes an openloop sequence of controls optimizing the future behaviour, and applies the first action of this sequence. Using measurements to update the optimization problem for the next time step introduces feedback. An asset of MPC is the easy handling of constraints. Other potential benefits of MPC in power system control problems have been demonstrated e.g. in [8], [9], [10].

The paper is organized as follows. The proposed method is described in Section II. A simple but detailed example is given in Section III. Concluding remarks are given in Section IV.

\section{THE PROPOSED APPROACH}

\section{A. Objective}

As outlined in the Introduction, a multi-step optimization closely inspired of MPC is proposed to determine a sequence of actions aimed at alleviating line overloads. More precisely, the objective is to bring the currents in overloaded transmission lines below their admissible values before they are taken out 
of service, which may trigger cascading failures. The actions are taken over a finite number of time steps, compatible with the tolerable overload duration.

Furthermore, limits on the rate of change of controls are taken into account, which may lead to replacing a "cheap" but slow control with a more "expensive" but faster one.

Finally, by incorporating measurements gathered at each time step, and adjusting accordingly its next action, the MPC scheme operates in closed-loop mode until the overload has been eliminated, which allows to somewhat compensate for model inaccuracies [7].

\section{B. Modelling and statement of the problem}

A typical control sequence is depicted in Fig. 1. The proposed discrete controller acts at multiples of a period $\Delta t$. Assume that some line gets overloaded in the interval $\left[\begin{array}{ll}t_{0}-\Delta t & t_{0}\end{array}\right]$, thus causing the emergency condition to be detected at time $t_{0}$, and the controller to act for the first time at $t_{0}+\Delta t$. Let $T_{o l}$ be the duration the overload can be tolerated before the line is tripped. For security, a settling delay $M$ is left after the last control action and the time of overload occurrence is taken as $t_{0}-\Delta t$. Thus, the controller has to remove the overload in at most $K$ steps, where $K$ is the largest integer such that $(K+1) \Delta t \leq T_{o l}-M$.

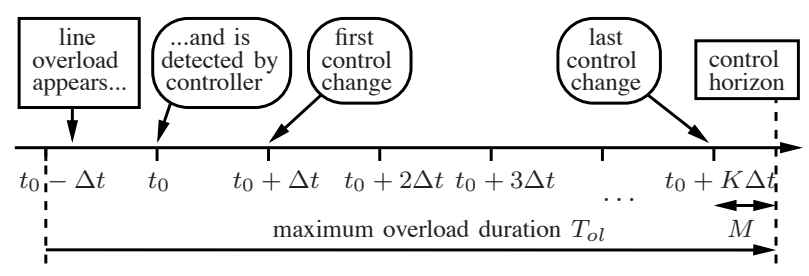

Fig. 1. Sequence of events and controls

Let us denote by $\Delta \mathbf{u}^{j}$ the vector of control changes applied at time $j \Delta t(j=1, \ldots, K)$. This may involve Phase Shifting Transformer (PST) angles, generator active powers and load powers.

The system model used in the controller is linear and obtained from a DC approximation of the actual power flows. This simplification is acceptable to the extent that in most real-life situations where cascade line tripping took place, the system was in normal operating conditions when the first line overload(s) appeared; large transients (such as interarea oscillations or frequency swings) appeared in an already degraded situation resulting from a significant number of line outages (leading in extreme cases to network split). Although better modelling is desirable, the closed-loop MPC scheme is expected to compensate for inaccuracies to some extent.

Under the above assumptions, the vectors of line (active power) flows at the successive times $(j-1) \Delta t$ and $j \Delta t$ are linked through:

$$
\mathbf{p}^{j}=\mathbf{p}^{j-1}+\mathbf{S} \Delta \mathbf{u}^{j}
$$

where $\mathbf{S}$ is the sensitivity matrix of branch power flows to controls. This matrix is easily derived from the DC load flow Jacobian. It can be computed row-by-row or column-bycolumn using a well-known sensitivity formula. With standard sparsity techniques, this computation is efficient even in the case the controller would monitor a large region. If the DC approximation is used, this matrix has to be updated only after a change in topology.

The control objective is to have, at step $K$, the flow in the overloaded line brought back within its admissible limits, and no new line overloaded. This is written as:

$$
-\mathbf{p}^{\max } \leq \mathbf{p}^{K} \leq \mathbf{p}^{\max }
$$

where $\mathbf{p}^{\max }$ is the vector of branch power flow limits.

\section{Multi-step optimization}

At time $t_{0}$, a sequence of $K$ future controls $\left(\Delta \mathbf{u}^{1}, \Delta \mathbf{u}^{2}, \ldots, \Delta \mathbf{u}^{K}\right)$ is computed in order to bring the power flows from their initial value $\mathbf{p}^{0}$ to a value satisfying (2). This sequence is computed so as to minimize the total "cost" associated with control changes, while keeping the rate of change of the latter within the allowed limits.

The sequence of $K$ future controls is thus obtained as the solution of the optimization problem:

$$
\min _{\Delta \mathbf{u}^{1}, \Delta \mathbf{u}^{2}, \ldots, \Delta \mathbf{u}^{K}} \sum_{j=1}^{K} \sum_{i=1}^{n} d_{i}^{j} c_{i}\left|\Delta u_{i}^{j}\right|
$$

$$
\begin{array}{cc}
\text { subject to: } \quad \mathbf{p}^{j}=\mathbf{p}^{j-1}+\mathbf{S} \Delta \mathbf{u}^{j} & j=1, \ldots, K(4) \\
\Delta \mathbf{u}^{\text {min }} \leq \Delta \mathbf{u}^{j} \leq \Delta \mathbf{u}^{\max } & j=1, \ldots, K(5) \\
\mathbf{u}^{j}=\mathbf{u}^{j-1}+\Delta \mathbf{u}^{j} & j=1, \ldots, K(6) \\
\mathbf{u}^{\text {min }} \leq \mathbf{u}^{j} \leq \mathbf{u}^{\text {max }} & j=1, \ldots, K(7) \\
-\mathbf{p}_{\text {mo }}^{\text {max }} \leq \mathbf{p}_{\text {no }}^{j} \leq \mathbf{p}_{\text {mo }}^{\text {max }} & j=1, \ldots, K(8) \\
-\mathbf{p}^{\text {max }} \leq \mathbf{p}^{K} \leq \mathbf{p}^{\text {max }} &
\end{array}
$$

In the objective function (3), $c_{i}$ is a cost associated with the $i$-th control change $\Delta u_{i}, d_{i}^{j}$ is a "discount factor" used to weight the cost of this control at the $j$-th time step, and $n$ is the total number of controls.

Equation (4) represents a time sequence of linear predictions of the type (1). $\Delta \mathbf{u}^{\max }$ in (5) is obtained by multiplying the maximum rate of increase of each control by the time interval $\Delta t$, and similarly for $\Delta \mathbf{u}^{\min }$. The constraints $(6,7)$ obviously aim at keeping the controls within their admissible limits, corresponding to $\mathbf{u}^{\min }$ and $\mathbf{u}^{\max }$, respectively.

In the inequality (8), $\mathbf{p}_{n o}^{j}$ is a subvector of $\mathbf{p}^{j}$ corresponding to the initially non overloaded lines. The objective is to prevent lines that are initially within their limits from getting subsequently overloaded by the controller (although not mandatory, this choice is made for the sake of security).

Other objectives than (3) can be thought of, for instance the quadratic one:

$$
\min _{\Delta \mathbf{u}^{1}, \Delta \mathbf{u}^{2}, \ldots, \Delta \mathbf{u}^{K}} \sum_{j=1}^{K} \sum_{i=1}^{n} c_{i}\left(\Delta u_{i}^{j}\right)^{2}
$$

which tends to distribute the control changes more evenly over the time window.

The above multi-step optimization relies on the vector $\mathbf{p}^{0}$ of branch power flow measurements, gathered at time $t_{0}$, and the initial value $\mathbf{u}^{0}$ of controls. According to MPC principle, 
only the first control step $\Delta \mathbf{u}^{1}$ of the so computed sequence is applied, at time $t_{0}+\Delta t$. At that time, new measurements are collected in $\mathbf{p}^{1}$ and a completely new control sequence is computed for the next time steps [7].

Note that power flow measurements can be collected at a higher frequency than controller actions. This is probably desirable to filter out transients and focus on the long-term trend of the power flows.

A noteworthy feature of the proposed algorithm is the possibility of dynamically updating the value of $T_{o l}$ and hence the number $K$ of control steps. Indeed, as the controller starts alleviating line overloads, more time is available before the lines trip (provided the overcurrent protections have not been designed with a single, fixed temporization $T_{o l}$ ). This allows replacing expensive fast emergency controls with slower but cheaper ones. This extension of the control window leaves more time for the controller to compensate for modelling errors.

\section{Simulation RESUltS}

\section{A. Test system}

In this section, an illustrative example is presented in detail, based on the academic system shown in Fig. 2.

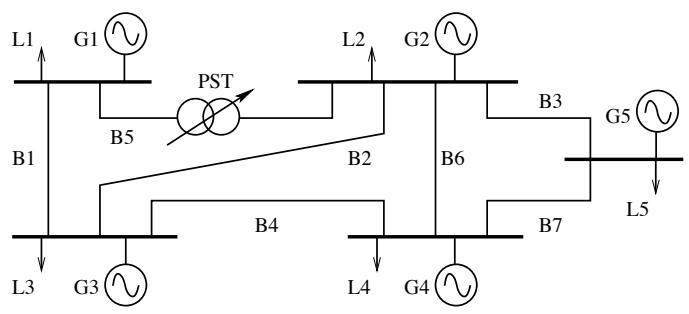

Fig. 2. System used in illustrative example

For the sake of illustration, we assume $\Delta t=5 \mathrm{~s}$, which could be representative of a real implementation.

Due to some disturbance, lines B1 and B6 get overloaded at time $t=2 \mathrm{~s}$. This is first noticed by the controller at $t_{0}=$ $5 \mathrm{~s}$ (see Fig. 1). A fixed overload duration $T_{o l}=60 \mathrm{~s}$ is assumed and the settling delay $\mathrm{M}$ is set to $\Delta t=5 \mathrm{~s}$. Hence the controller has to relieve the overloads in at most $K=$ $(60-5-5) / 5=10$ steps.

Note that this is a stringent test in which the control horizon is not receding. Thus, $K$ decreases from 10 to 1 in the successive application of the MPC algorithm. The number of constraints (4-8) decreases accordingly as time goes.

The available controls are the angle of the PST in branch B5, the production of generators G1 and G5 and the power of the interruptible load L3. Through the relative values of the costs $c_{i}$, priority is given to actions on the PST, then on the generators and finally on the load, for obvious reasons. Constraints are imposed on the changes in PST angle, power generations and load power that can take place in a single control step. The available controls, their costs and their bounds are given in Table I.
TABLE I

AVAILABLE CONTROLS, COSTS AND BOUNDS

\begin{tabular}{|c|c|c|c|}
\hline control & $c_{i}$ & $\Delta u^{\text {min }}$ & $\Delta u^{\max }$ \\
\hline PST & 1 & -1 deg. & 1 deg. \\
gener. G1 and G5 & 10 & $-2 \mathrm{pu}$ & $2 \mathrm{pu}$ \\
load L3 & 100 & 0 & $2 \mathrm{pu}$ \\
\hline
\end{tabular}

\section{B. Simulations based on exact model}

This subsection illustrates the behaviour of the proposed controller when its model matches the system behaviour exactly. To this purpose, the matrix $\mathbf{S}$ of the real system has been obtained by linearization around the current operating point. Then, this matrix has been used both to simulate the system response to the controller and in the controller itself. Under these ideal conditions, the behaviour of various objective functions is compared.

We first consider the absolute-value objective (3). Figure 3 shows the time evolution of power flows and controls, respectively. All transmission lines have the same limit, shown with the dash-dotted horizontal lines in the upper plot. As can be seen, the two initial overloads are removed, while the other lines are kept within limits. The controller uses the cheap PST to the greatest possible extent. Acting on the PST reduces the power flow in line B6 but increases the one in line B1. Therefore, the controller subsequently uses the more expensive generation rescheduling. This alleviates the above two lines but increases the flow in line B7. Finally, load shedding is used because the problem cannot be solved with the sole help of PST and generators.
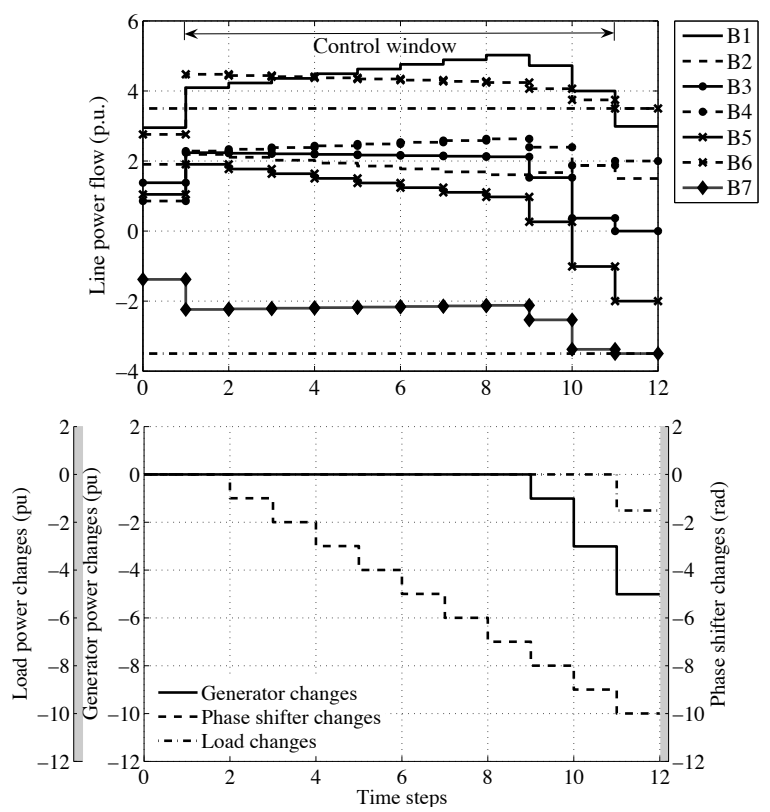

Fig. 3. Line flows and control actions with absolute-value objective

In the previous example, the discount factors $d_{i}^{j}$ were chosen to favour actions taken at the end of the control window, and the same factors were used for all types of controls, as illustrated with heavy line in Fig. 4. Alternatively, different control types can be assigned different discount factors in 
order, for instance, to favour early actions on generators and later actions on load shedding. Furthermore, the discount factors can be set so that overloads are corrected earlier in the control window. In this case, some time is left for the controller to apply additional corrections, not anticipated in the first control steps but required due to measurement noise or model inaccuracies, for instance.

An example is given in Fig. 5 where the discount factors favour PST and generators actions around the 2nd control step (applied at 3rd time step) and load shedding around the 8th control step (as shown with dashed line in Fig. 4). A comparison with Fig. 3 shows that generation is indeed rescheduled earlier. However, this action is inhibited at the 4th time step by the fact that (the previously non overloaded) line B7 is approaching its limit. A second rescheduling takes place later on, at the same time as load shedding.

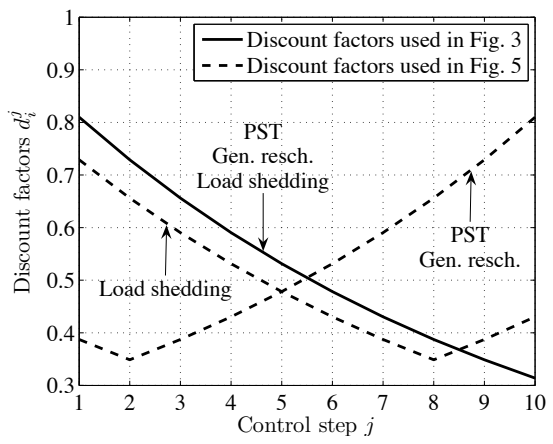

Fig. 4. Discount factors
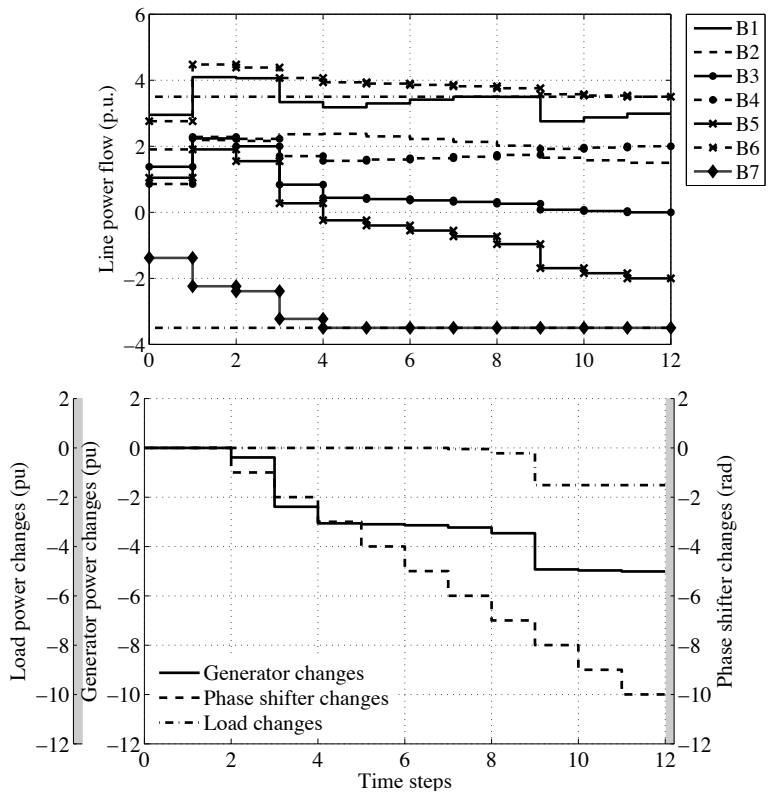

Fig. 5. Same case as in Fig. 3 with different discount factors

While the absolute-value objective (3) was used in the examples presented so far, the results shown in Fig. 6 were obtained with the quadratic objective (10) (without discount factors). As expected, the control actions are equally distributed over the control window.

This objective is used in the remaining of the paper.
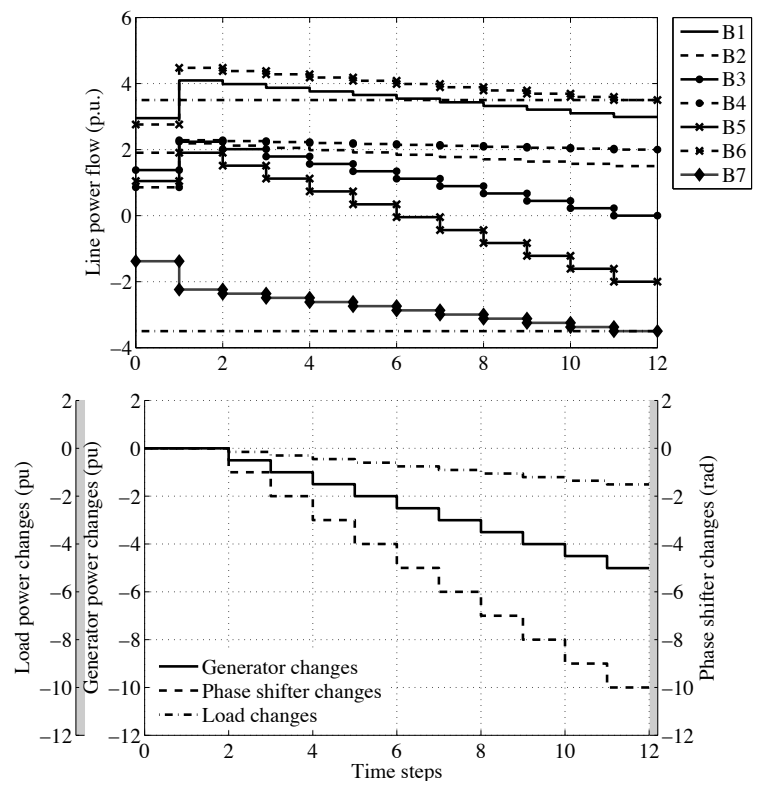

Fig. 6. Line flows and control actions with quadratic objective

\section{Simulations with approximate controller model}

A realistic test of the proposed method requires to consider the effect of model inaccuracies and measurement noises.

To deal with the first aspect, the system response was simulated with the reference matrix $\mathbf{S}$ while random errors were introduced on the reactances of (overloaded) lines B1 and B6 before building the $\mathbf{S}$ matrix used by the controller. The random error was uniformly distributed in the interval $\left[\begin{array}{ll}-0.2 X & 0.2 X\end{array}\right]$ where $X$ is the reactance of the line of concern.

Under the effect of these random errors, one may expect the model used by the controller to be either pessimistic or optimistic with respect to the real system behaviour. These two situations are illustrated in Figs. 7 and 8, respectively. Both should be compared to Fig. 6 which was obtained when using the exact model in the controller.

In the case of Fig. 7, it can be seen that generator rescheduling and load shedding decrease with time because the controller senses that the situation is improving faster than initially expected. Figure 8, on the other hand, shows a case where the model available to the controller is too optimistic. This leads to control actions increasing with time, as the situation is not improving as anticipated. In this case, the initial overload of line B6 is not alleviated at the end of the control window, while line B7 gets overloaded as a result of the control actions.

In order to estimate the failure rate of the controller, 5000 Monte Carlo simulations were run, involving the above mentioned random errors.

The statistical distributions of power flows in branches B1 and B6 at the end of the control window are shown in Fig. 9. The thermal limit corresponding to the dashed vertical line, one can see that the algorithm failed to eliminate the overload in line B6 in more than half of the cases. This means that the 

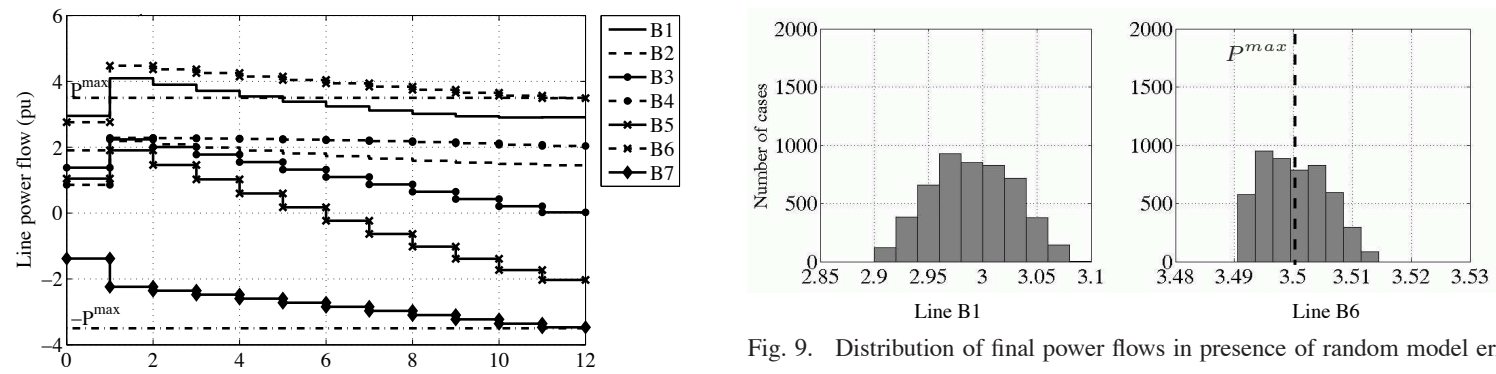

Fig. 9. Distribution of final power flows in presence of random model errors

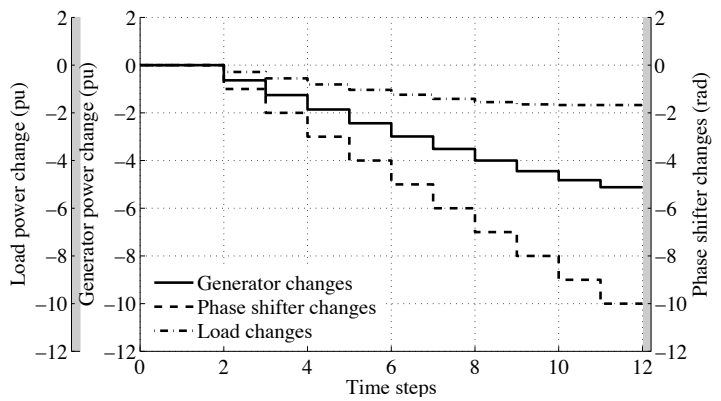

Fig. 7. Line flows and control actions; model used by controller is pessimistic
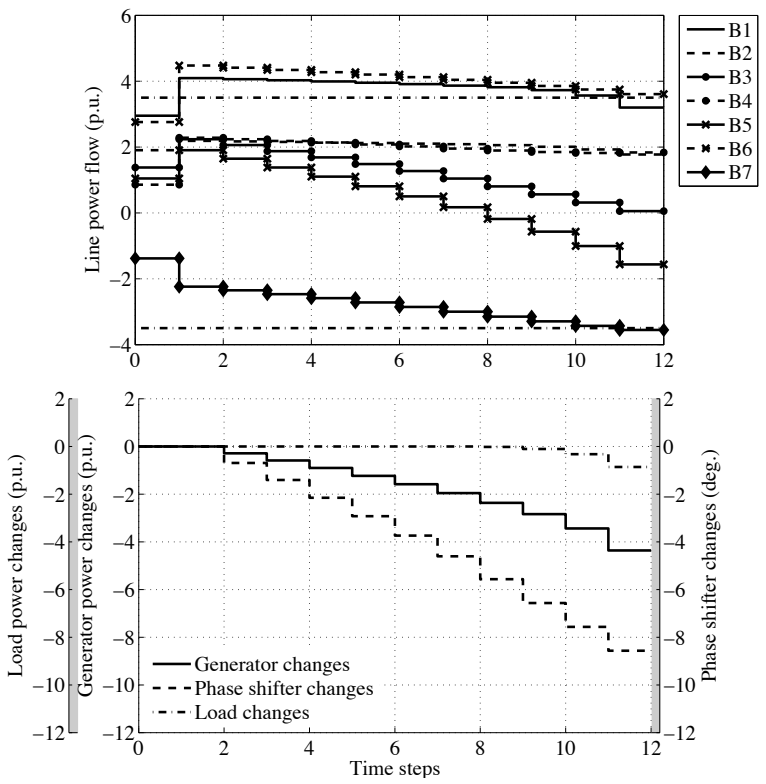

Fig. 8. Line flows and control actions; model used by controller is optimistic

controller could not prevent line B6 from being tripped.

In the presence of modelling errors, it takes more time to the MPC scheme to reach its objective. If the control window was updated with time, i.e. extended in response to the observed decrease in line currents, the controller would be given more chances to meet its target.

\section{Simulations incorporating noisy measurements}

Another source of inaccuracy is the noise affecting the power flow measurements gathered at each control step.

In the case of Fig. 10, an identical error was added to each component of the successive $\mathbf{p}^{j}$ vectors. In the case of Fig. 11,
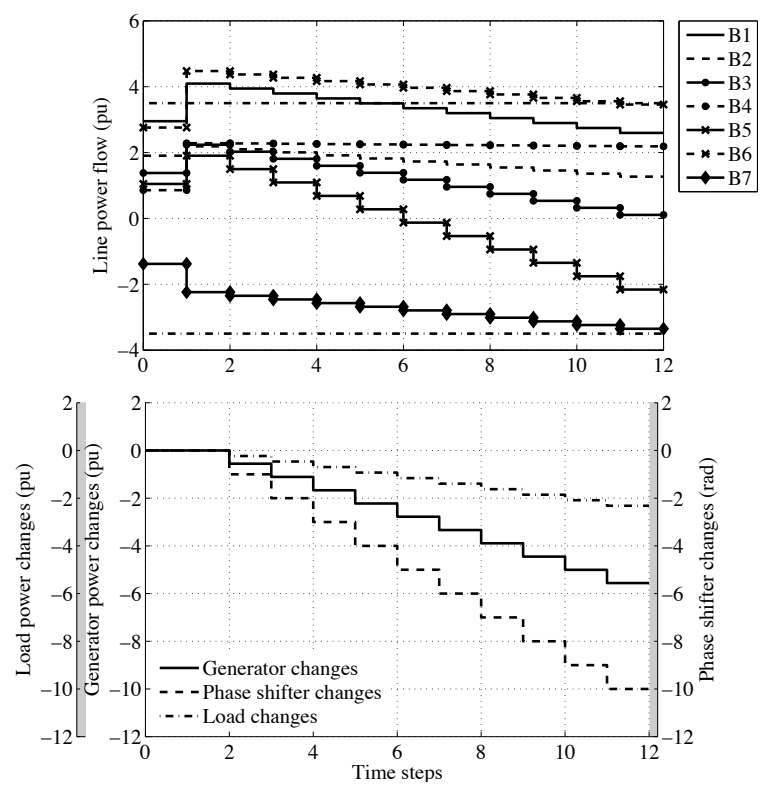

Fig. 10. Line flows and control actions; constant measurement error on power flows (the flows shown are the real ones)

the noise was a random variable uniformly distributed in the interval $\left[\begin{array}{ll}-0.3 & 0.3\end{array}\right] \mathrm{pu}$. In both cases, the MPC algorithm adjusts its control sequence and succeeds removing the line overloads.

Although the controller acted successfully in both cases, there is a risk of failure especially if the measurement noise becomes too high. In this case, and the case from the previous section, the technique discussed hereafter might prove useful.

\section{E. Correction of errors in the MPC scheme}

As mentioned in the previous subsections, the MPC algorithm may fail reaching its target due to modelling errors or measurement noise, causing the overloads to be alleviated too late, especially in the case of a fixed overload duration $T_{o l}$. The problem is likely to be less severe if more controls (i.e. more degrees of freedom) are available to relieve the line overloads.

One way of counteracting this problem consists of setting the limit $P^{\max }$ of the initially overloaded lines to a smaller value - say $P^{l i m}$ - in order to cause the MPC algorithm to act more strongly, and hence the line power flows to come back faster below their limits. To this purpose, the limits $\mathbf{P}_{\text {no }}^{\max }$ of the initially non overloaded lines are left unchanged. If, at a given step, the power in an initially overloaded line becomes 

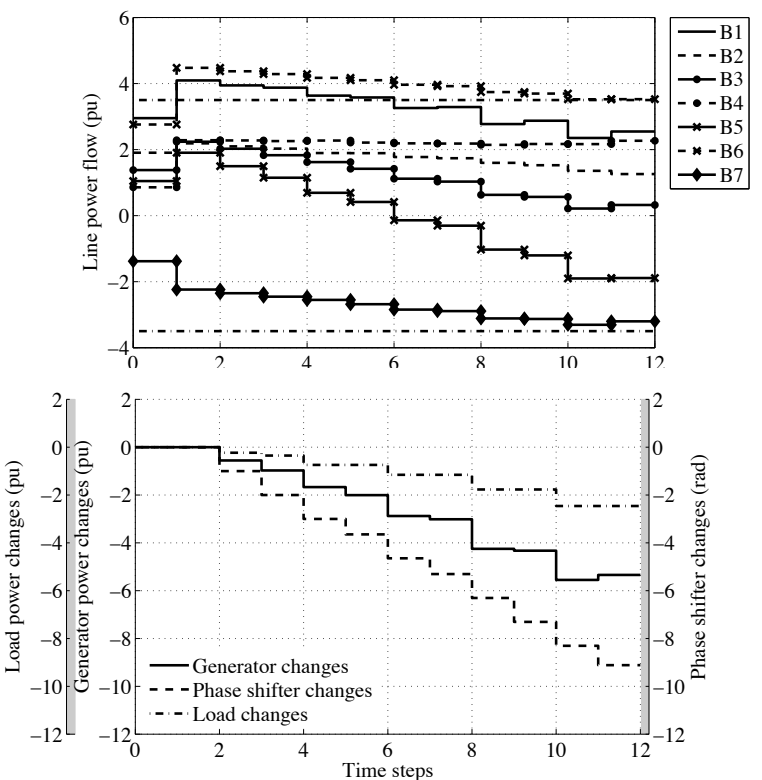

Fig. 11. Line flows and control actions; random measurement errors on power flows (the flows shown are the real ones)

smaller than $P^{\max }$, this line is moved to the non-overloaded category, even if its power flow remains larger than $P^{l i m}$.

The above correction makes the controller operate a bit conservatively, but this is fully acceptable for a system protection scheme aimed at acting in rare emergency conditions.

The simplest scheme consists of choosing $P^{\text {lim }}$ a priori. Monte-Carlo analysis can be used to this purpose. For instance, Figure 12 shows the statistical distribution of final power flows over the set of 5000 cases previously considered in Fig. 9. The artificial limit $P^{\text {lim }}$ has been set to $3.48 \mathrm{pu}$, which represents only a small decrease with respect to the real limit. As shown by the histogram, this was enough for the controller to operate successfully in all 5000 cases.
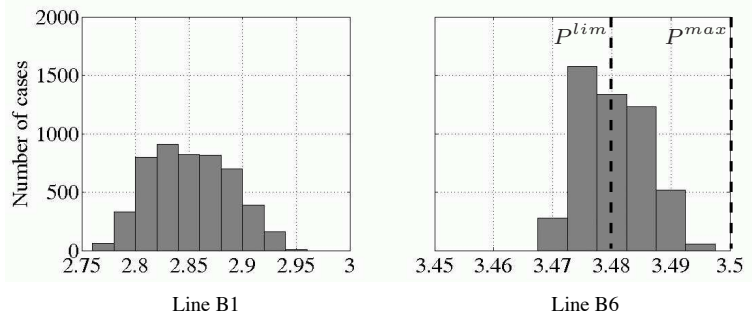

Fig. 12. Distribution of final power flows; same cases as in Fig. 9 using $P^{\text {lim }}$ as line limit in the optimization

As an alternative, $P^{l i m}$ could be adjusted dynamically, when the overload reduction is smaller than expected. For instance, one may subtract from $P^{l i m}$ the difference between the measured and the expected line power flow, divided by the number of remaining steps in the control window. This scheme has been found to work satisfactorily on the test system, but has to be tested more carefully with a more realistic model taking into account the presence of other controls.

\section{CONCLUSION}

Cascade transmission line trippings due to thermal overload may lead to blackouts. System protection schemes against these events are rare, in particular because they require to handle a real-time model of the system.

The scheme proposed in this paper, acts to bring line currents below their limits before they are tripped. It is inspired of MPC, and operates in closed-loop in the sense that, at each time step new measurements are used to update a multistep optimization problem, and hence cope with the new prevailing conditions. Various control actions can be included in the objective function with different priorities, and various objectives can be considered.

Another feature of the proposed scheme is the capability to update the control horizon in the course of applying the actions. Thus, as transmission lines get alleviated, more time is left to act, i.e. more control steps become available.

The closed-loop nature of the control guarantees some robustness with respect to modelling errors and measurement noises. However, they may lead the controller to take more time to remove the overload. This may be critical in the case where the overload duration is fixed (i.e. not updated with the improving conditions) and when a limited number of controls are available. This can be compensated by reducing, statically or perhaps dynamically, the limits assigned to overloaded transmission lines in the optimization problem.

Clearly, additional aspects have to be investigated, such as measurement filtering, the choice of objective functions, interactions with other controllers, improved dynamic compensation of modelling errors, etc. As of writing this paper, successful results have been obtained on a real-life system.

\section{REFERENCES}

[1] S. Talukdar, D. Jia, P. Hines, B.H. Krogh, "Distributed model predictive control for the mitigation of cascading failures", Proc. 44th Conf. on Decision and Control and European Control Conference, CDC-ECC 2005, Dec. 2005.

[2] D. H. Karlsson, convener, "System Protection Schemes in Power Networks". Final report of CIGRE Task Force 38.02.19

[3] O. Alsac, B. Stott, "Optimal power flow with steady-state security", IEEE Trans. on PAS, Vol. PAS-93, 1974, pp. 745-751.

[4] J. A. Momoh, J. Z. Zhu, G. D. Boswell, S. Hoffman, "Power System Security Enhancement by OPF with Phase Shifters", IEEE Trans. on Power Systems, Vol. 16, 2001, pp. 287-293. 4. J. A. Momoh, B. J. Koessler,

[5] M. S. Bond, B. Stott, D. Sun, A. Papalexopoulos, P. Ristanovic, "Challenges to Optimal Power Flow", IEEE Trans. on Power Systems, Vol. 12, 1997, pp. 444-455.

[6] K.R.W. Bell, A.R. Daniels, R.W. Dunn, "Alleviation of transmission system overloads using fuzzy reasoning", Fuzzy Sets and Systems (Elsevier), Vol. 102, 1999, pp. 41-52.

[7] J.M. Maciejowski, Predictive control with constraints, Prentice Hall, Harlow (England), 2002, ISBN 0201398230.

[8] M. Zima, G. Andersson, "Model predictive control of electric power systems under emergency conditions", In Real-time stability in power systems: Techniques for early detection of the risk of blackouts, Springer, pp. 273-292, 2006.

[9] N. Atic, D. Rerkpreedapong, A. Hasanovic, A. Feliachi, "NERC compliant decentralized load frequency control design using model predictive control", Proc. IEEE PES General Meeting, Toronto, vol. 2, 2003.

[10] I.A. Hiskens, B. Gong, "Voltage stability enhancement via model predictive control of load", Intelligent Automation and Soft Computing, vol. 12, 2006, pp. 117-124. 